\author{
Araştırma Makalesi / Research Article
}

\title{
Uzaktan Algılama Görüntülerinin Evrişimsel Sinir Ağları ve Komşuluk Bileşen Analizi Tabanlı Özniteliklerinin Sınıflandırıması
}

\author{
Fatih ÖZYURT \\ FIrat Üniversitesi Enformatik Bölümü 23119 Elazığ \\ e-posta: fatihozyurt@firat.edu.tr ORCID ID: https://orcid.org/0000-0002-8154-6691
}

Geliş Tarihi: 26.04.2019; Kabul Tarihi: 05.12.2019

\begin{tabular}{cl} 
& Öz \\
\cline { 2 - 3 } & Bu çalışmada, WHU-RS19 veri setinden elde edilen uzaktan algılama görüntülerinin sınıflandırması için \\
farklı derin öğrenme modellerinden alınan özniteliklerin komşuluk bileşen analizi ile indirgenip Destek \\
Anahtar kelimeler & Vektör Makinesi (DVM) ile sınıflandırması yapılmışır. WHU-RS19 veri setinin görüntüleri ESA \\
Derin Öğrenme; & modellerinden AlexNet, VGG-16 ve GoogleNet'e girdi olarak verilmişve her bir mimarinin son tam bağlı \\
Evrişimsel Sinir Ağ; & katmanından 1000'er adet öznitelik elde edilmiştir. Ayrıca üç mimariden elde edilen öznitelikler \\
Destek Vektör & birleştirilerek komşuluk bileşen analizi (KBA) yöntemiyle 1000 özniteliğe indirgenmiştir. Aynı veriyi \\
Makinesi; & kullanan diğer çalışmalar ile kıyaslama yapılabilmesi için mevcut verilerin \%60 ve \%40'ı kullanılarak \\
Komşuluk Bileşen & eğitimi DVM ile gerçekleştirilmiştir. Çalışma kapsamında KBA ile özniteliği indirgenmiş verilerin \%60'ı \\
Analizi & eğitim olarak kullanıldığında \%98.75, \%40'ı eğitim olarak kullanıldığında ise \%97.01 oranında başarım \\
& elde edilmiştir. Bu başarım oranları mevcut çalışmalara göre daha üstün performans sağladığı \\
& görülmüştür.
\end{tabular}

\section{Classification of Remote Sensing Images Based on Convolutional Neural Networks and Neighborhood Component Analysis Features}

\begin{abstract}
Keywords

Deep Learning; Convolutional Neural Network;

Support Vector Machine;

Neighborhood Component Analysis

In this study, for the classification of the images obtained from the WHU-RS19 dataset, the features obtained from different deep learning models were reduced by neighboring component analysis (NCA) and classified with the Support Vector Machine (SVM). The images of the WHU-RS19 data set were given as input to the CNN models AlexNet, VGG-16 and GoogleNet and 1000 features were obtained from the last fully connected layer of each architecture. Furthermore, the features obtained from the three architectures were combined and reduced to 1000 features by NCA method. In order to make comparisons with other studies that use the same data, $60 \%$ and $40 \%$ of the existing data were trained with SVM. In the study, when the $60 \%$ of the data were used as training $98.75 \%$ accuracy obtained. When the $40 \%$ of the data were used as training \%97.0.1 accuracy obtained. It has been found that superior performance compared to current studies.
\end{abstract}

(C) Afyon Kocatepe Üniversitesi

\section{Giriş}

Havadan alınan görüntüler, Dünya'nın yüzeyini ayrıntılı bir şekilde ölçmemize olanak sağlaması açısından büyük önem taşıyan bir tür veri kaynağıdır (Hu et al. 2013, Cheng et al. 2015, Cheng et al. 2014, Hu et al. 2015). Hava görüntülerinin karmaşık geometrik yapıları ve mekânsal desenleri nedeniyle anlamsal içeriğini etkin bir şekilde dünyadaki birçok uygulama tarafından anlamlandırılması özellikle önemlidir. Çünkü bu görüntüler coğrafi bilgi sistemlerinin kullanımı açısından önem arz etmektedir. Kamu ve özel sektördeki birçok kurum tarafından coğrafi bölgelerin ve coğrafi alanların (orman, bina, arazi vb.) ayrılmasında kullanılmaktadır. Günümüzde, uzaktan algılayıcı görüntülerin işlenmesi ile ilgili artan bir ilgi vardır (Çalışkan 2018, Göksu et al. 2018, Mutlu 2018). Algoritmalardaki ve bilgisayar donanımındaki gelişmeler uzaktan algılama verilerinin işlenmesinde önemli katkılar sağlamıştır. 
Derin öğrenmenin gerçek zamanlı nesne izleme ve sınıflandırmadaki başarısı, akademik ve endüstriyel çevreleri derin öğrenme alanında araştırma yapmaya yönlendirmiştir. ESA mimarisi, nesnelerin tespiti ve nesnelerin sınıflandırılması için çok başarılıdır (Özyurt et al. 2019, Zhang et al. 2019, Yang et al. 2019, Huang et al. 2019).

ESA, etiketli görüntülerden öznitelik çıkarabilen çok katmanlı derin öğrenme mimarilerinden biridir. Görüntü, ses analizi, robot teknolojisi, uzaktan algılama, genetik analiz, tıbbi hastalık teşhisi gibi birçok alanda kullanılmaktadır. Bu çalışmada akademik çalışmalarda sıklıkla kullanılan AlexNet, VGG-16 ve GoogleNet gibi ESA mimarileri kullanılmıştır. Bu ESA mimarileri, farklı çalışma grupları tarafından geliştirilen önceden eğitilmiş ESA modelleridir. Alex Krizhevsky tarafından tasarlanan Alexnet mimarisi, 2012 yılında ImageNet yarışmasını kazandı (Alom, Taha, Yakopcic, Westberg, Sidike, Nasrin, Asari, 2018). Alexnet, bir milyonun üzerinde görüntü ile eğitilmiş yüksek başarı oranına sahip, derin bir öğrenme mimarisidir. Google Net, ImageNet-2014 resim sınıflandırma yarışmasında ilk sırada yer almıştır (Han, Zhang, Cheng, Liu, Xu, 2018) . 2012 yılında 89.06\% başarı oranı GoogleNet mimari ile 93.33\% oranına yükselmiştir. VGG-16 mimarisi GoogleNet mimarisinden sonra ikinci olmuştur (Qassim, Verma, Feinzimer, 2018). Sınıflandırılacak her ver setinin eğitimi için büyük veri setlerine ihtiyaç duyulmaktadır. Böyle durumları aşmak için önceden eğitilmiş bir mimari kullanmak daha iyi olacaktır. Bu durumu ifade eden yapı Transfer Öğrenimi olarak adlandırılmaktadır (Khan, Islam, Jan, Din, Rodrigues, 2019) . Yeni bir ESA mimarisi ağı eğitmek için yeterli veri yoksa özellik vektörü önceden eğitilmiş ESA mimarileri kullanılarak çıkarılabilir. Bu çalışmada, WHU-RS19 veri setinden elde edilen görüntülerin önceden eğitilmiş ESA mimarileri olan AlexNet, GoogleNet ve VGG-16 mimarileri kullanılarak özniteliklerin çıkartıldığı ve Kübik DVM ile sınıflandırıldığı bir yöntem önerdik (Arel et al. 2010). WHU-RS19 veri kümesi görüntüleri, AlexNet, GoogleNet ve VGG-16 mimarilerinin giriş katmanına verilmek için mimarilere uygun boyutlara dönüştürüldü. Öznitelikler, AlexNet mimarisinin Fc8, GoogleNet mimarisinin loss3-classifier ve VGG-16 mimarisinin Fc8 katmanlarından elde edilmiştir. AlexNet, GoogleNet ve VGG-16 mimarilerinin ilgili katmanlarından sırasıyla, 1000'er öznitelik elde edilmiştir. Çıkarılan özellikler kübik DVM ile sınıflandırılmıştır. Daha sonra 3 mimariden elde edilen öznitelikler birleştirilerek 3000 öznitelik KBA algoritmasıyla 1000 adet özniteliğe indirgenip kübik DVM ile sınıflandırımıştır. KBA algoritmasıyla verimli özniteliklerin seçildiği görülmüştür. Sınıflandırma başarımları aynı veri seti kullanan diğer çalışmalar ile kıyaslanmıştır.

\section{Materyal ve Metot}

Bu çalışmanın amacı, eğitilmiş ESA mimarilerinden AlexNet, GoogleNet ve VGG-16 ile transfer öğrenme yöntemini kullanarak öznitelikleri çıkartıp bu öznitelikleri KBA öznitelik indirgeme algoritmasıyla indirgemektir. KBA algoritması ile toplamda elde edilen 3000 öznitelik 1000 özniteliğe indirgenmiştir. Elde edilen verimli öznitelikler en yüksek başarımı verdiği için kübik DVM ile sınıflandırılmıştır. Ek olarak, ESA'yı eğitmek için kullanılan geri yayılma algoritması çok yavaştır ve birçok hiperparametreyi ayarlamak gerektirir (Özyurt 2019). Bu zayıflıkların üstesinden gelmek için, ESA öznitelikleri çıkartılarak uzaktan algılanmış görüntülere uygun olan Destek Vektör Makinesi tabanlı (DVM) temelli yeni bir yaklaşım sunulmaktadır.

Bu makalede, çevrimiçi kullanıma açık farklı sahne sınıflarından çok çeşitli uzaktan algılama görüntülerini kapsayan bir veri seti kullanmaktayız. WHU-RS19 veri seti, havaalanı, plaj, köprü, ticari alan, çöl, tarım arazileri, futbol sahası, orman, sanayi bölgesi, çayır, dağ, park, park yeri, gölet, liman, tren istasyonu, konut, alan, nehir ve viyadük dahil olmak üzere 19 sahne sınıfından oluşmaktadır (Arel et al. 2010, Lv et al. 2014). Veri kümesinin tamamında 1.005 görüntü içermekte ve her sınıf için yaklaşık 50 görüntü vardır. Görüntü boyutları $600 \times 600$ pikseldir. WHU-RS19 veri kümesi ayrıca farklı sınıflandırma yöntemlerini hesaplamak için geniş çapta kabul edilmiştir. WHU-RS19 veri kümesine ait örnek gösterimler şekil 1'de verilmiştir. 

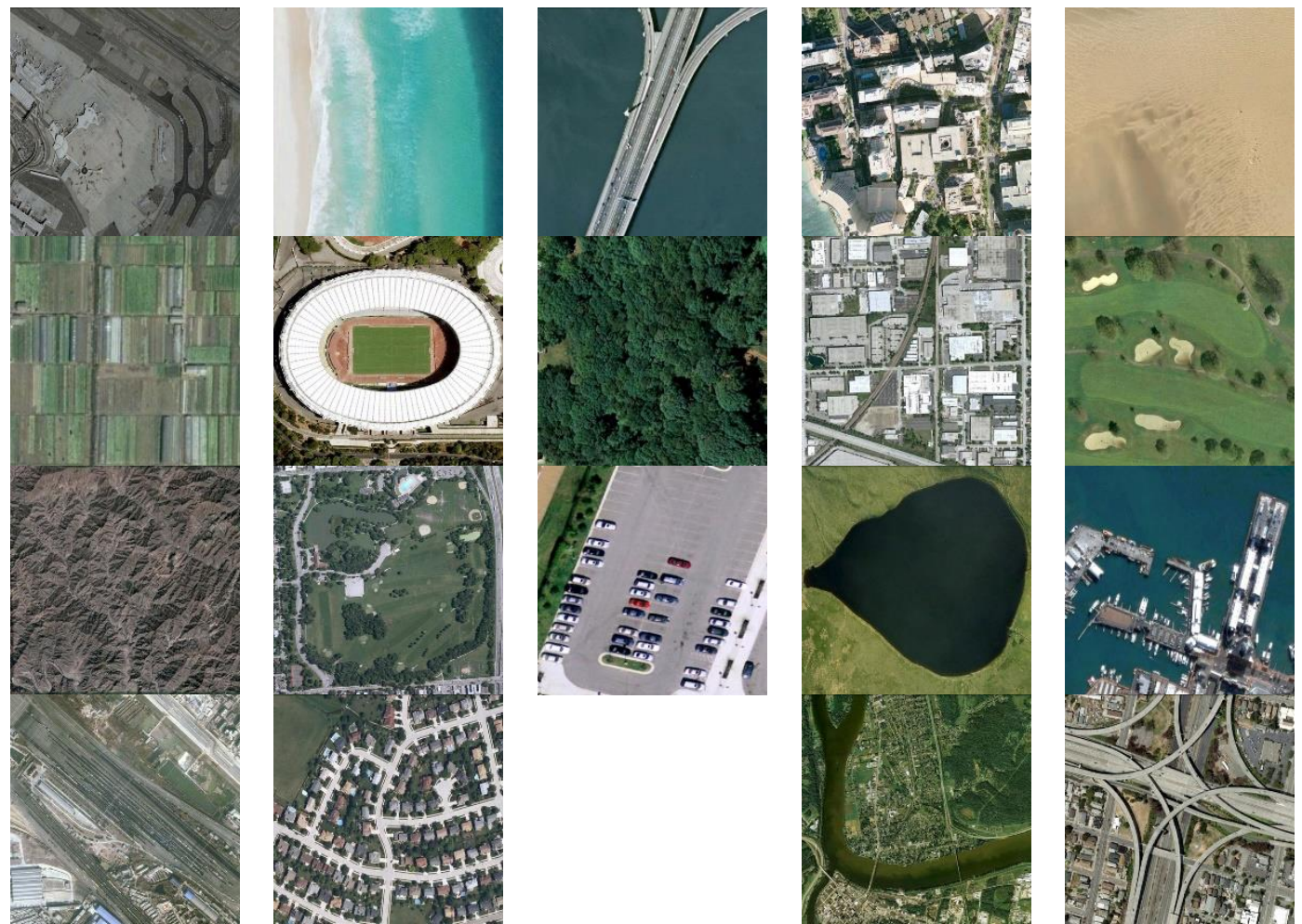

Şekil 1. WHU-RS19 verisetinden elde edilmiş örnek görüntüler

\section{1 Önceden Eğitilmiş ESA Mimarisi ile Öznitelik} Çıartma

Milyonlarca imge kullanarak eğitilmiş birkaç derin öğrenme mimarisi vardır. GoogleNet, VGG-16, AlexNet bunlara örnek olarak verilebilir. Bu derin öğrenme yöntemlerinin her biri, farklı mimariler kullanarak eğitim süreci gerçekleştirir. Bu çalışmada kullanılan derin öğrenme mimarileri AlexNet, GoogleNet ve VGG-16'dır. AlexNet, bir milyondan fazla resim kullanılarak eğitilmiş bir mimaridir (Krizhevsky et al. 2012). Ek olarak, bu mimari 1000 farklı kategoriye ayrılabilir. AlexNet mimarisinde toplamda 8 katmanlı bir yapıya sahip beş evrişim katmanı ve 3 tamamen bağı katman vardır. Evrişim katmanlarında farklı çekirdek boyutları $(11 \times 11,5 \times 5$, $3 \times 3)$ kullanılmaktadır. Evrişim katmanlarından sonra, maksimum havuzlama katmanı mevcuttur. VGG-16 mimarisi de milyonlarca resimle eğitilmiştir (Simonyan and Zisserman 2014). AlexNet gibi 1000 'in üzerinde farklı sınıfı ayırt edebilir. VGG-16, evrişim katmanlarında her zaman $3 \times 3^{\prime}$ lük filtreleri 1'er adımda, havuzlama katmanlarında ise $2 \times 2$ 'lik filtreleri 2'şer adım kaydırarak yapmaktadır. GoogleNet mimarisi ise derinlemesine 22 katmandan oluşmaktadır (Szegedy et al. 2015). Bu mimari parametre sayısını 60 milyondan (AlexNet) 4 milyona düşürmüştür. WHU-RS19 veri setindeki görüntülerin öznitelikleri, Alexnet mimarisinin Fc8 katmanından, GoogleNet mimarisinin loss3sınıflandırıc katmanından ve VGG-16 mimarisinin Fc8 katmanından çıkarılmaktadır. Bu mimarilerden 1000 'er adet öznitelik elde edilmiştir. Çalışmanın akış diyagramı Şekil 2'de verilmiştir.

\subsection{Komşuluk Bileşen Analizi (KBA) Algoritması}

Komşuluk Bileşen Analizinde aynı etiketi paylaşan noktaların komşularının farklı etiketlere sahip noktalardan daha dar olduğu bir alanı bulmak için en yakın komşulara benzer bir teknik kullanan bir algoritmadır. KBA'da özniteliklerin ağırlıkları, mesafe ölçümleri kullanılarak yapılmaktadır. KBA, parametrik olmayan, denetimli bir öznitelik seçim yöntemidir. Ayrıca Öklid mesafe ölçemeye alternatif mesafe ölçüm algoritmasıdır. KNN kullanılarak geliştirilmiş ve her öznitelik için pozitif ağırlıklar üretmektedir (Tuncer et al. 2019).

\subsection{Kübik Destek Vektör Makinesi}

DVM sınıflandırma tekniği, düşük bellek alanına sahip durumlarda kullanışlı bir sınıflandırıcıdır. DVM, sınıfları mümkün olan en iyi şekilde bölen çok boyutlu uzayda bir hiper düzlemi bulur (Lin et al. 2005). Bu makalede sınıflandırııının çekirdek fonksiyonunun denklem 1'de verilen kübik SVM tipi sınıflandırıcı kullanılmaktadır.

$$
K\left(x_{i}, x_{j}\right)=K\left(x_{i}^{\mathrm{t}}, x_{j}\right)^{3}
$$




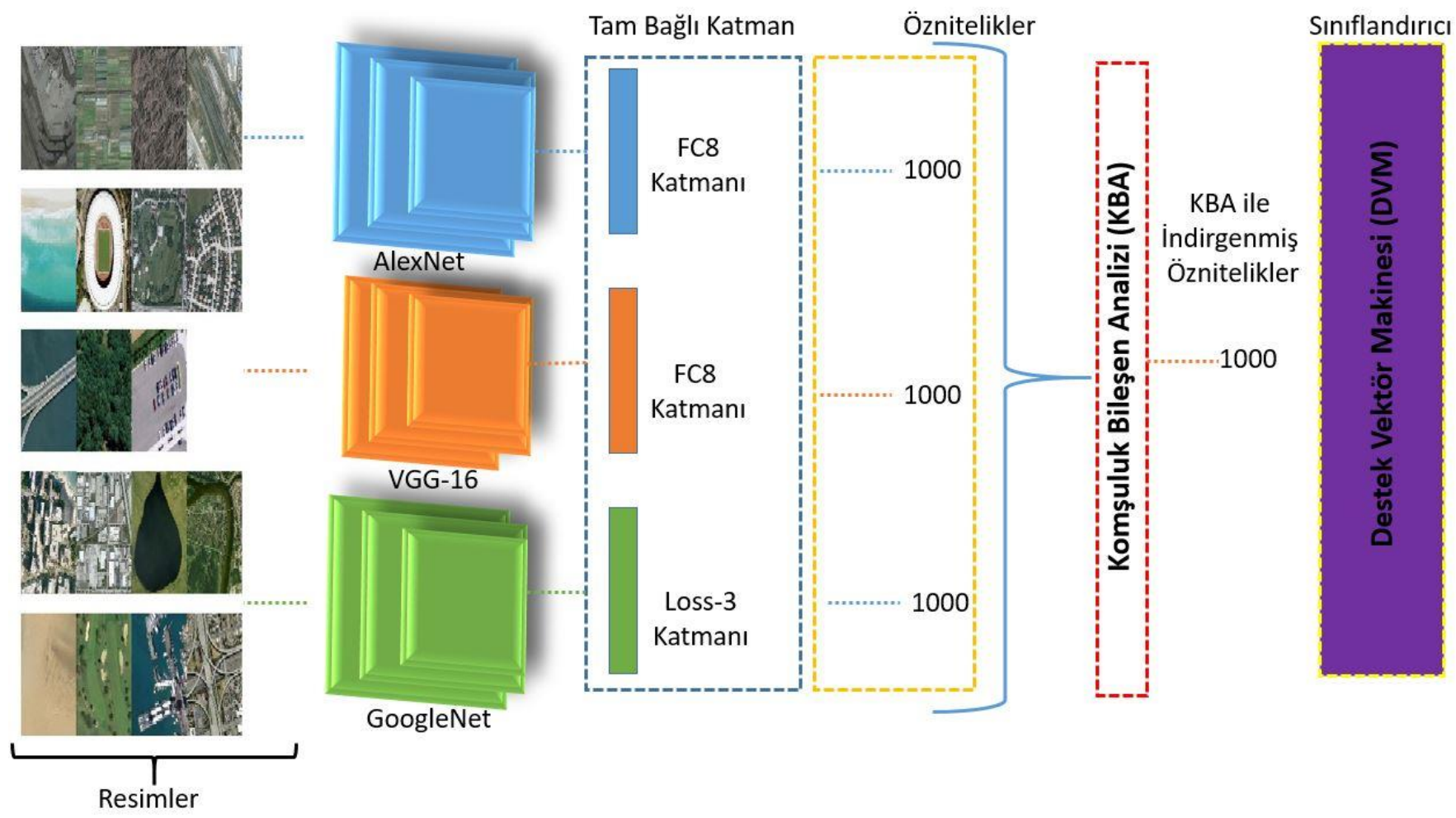

Şekil 2. Önerilen Yöntemin Akış Şeması

\section{Deneysel Sonuçlar ve Tartışma}

Öznitelik çıkarımı ve sınıflandırma için Intel Core i7 4510 işlemcili, 8 GB RAM ve Windows 10 işletim sistemine sahip bir dizüstü bilgisayar kullanılmıştır. Uygulama kodları MatConvnet Kütüphanesi kullanılarak MATLAB R2017a'da yazılmıştır. Deneysel değerlendirme 2 aşamadan oluşmaktadır. Birinci deneysel çalışmada her mimariden alınan öznitelikler $\% 60$ eğitim ve $\% 40$ eğitim verisi olacak şekilde ayarlandı. Ayrıca önerilen yöntemin güvenirliği için her eğitim 10'ar defa tekrar edildi. WHU-RS19 veri setinin görüntüleri ESA modellerinden AlexNet, VGG-16 ve GoogleNet'e girdi verileri olarak verildi. Çıkarılan öznitelikler kübik DVM ile sınıflandırılmıştır. ESA mimarilerinden elde edilen öznitelikler ile DVM sınıflandırıcısının doğruluk oranları Çizelge 1'de verilmiştir. Çizelge $1^{\prime}$ de görüldüğü üzere eğitim oranının artması başarım oranının direkt artmasını sağlamıştır.

Çizelge 1. ESA mimarisi ve DVM sınıflandırıcısının doğruluk oranları

\begin{tabular}{lcc}
\hline ESA Mimarisi & $\begin{array}{c}\text { \%60 oranında } \\
\text { Eğitim }\end{array}$ & $\begin{array}{c}\text { \%40 oranında } \\
\text { Eğitim }\end{array}$ \\
\hline AlexNet & $83.58 \pm 0.17$ & $78.77 \pm 0.01$ \\
\hline GoogleNet & $83.08 \pm 0.17$ & $79.60 \pm 0.14$ \\
\hline VGG-16 & $92.79 \pm 0.14$ & $88.23 \pm 0.12$ \\
\hline
\end{tabular}

GoogleNet ile AlexNet mimarileri yaklaşık sonuçlar verirken VGG-16 mimarisinin daha üstün performans sergilediği gözlemlenmiştir. Aynı veri setini kullanan diğer çalışmalar ile kıyaslama yapabilmek için eğitim verisi \%60'dan daha fazla arttırılmamıştır. İkinci deneysel çalışmasında 3 ESA mimarisinden elde edilen toplam 3000 öznitelik KBA algoritması kullanılarak 1000 özniteliğe indirgenmiştir. KBA öznitelik indirgeme algoritmasının verimli öznitelikleri seçebilme ve aynı veri setini kullanan diğer çalışmalara göre performansı analiz edilmiştir. íkinci deneysel çalışma sonuçları çizelge 2'de verilmiştir. 
Çizelge 2. KBA algoritması ile 1000 özniteliğe indirgenmiş WHU-RS19 veri setinin başarım oranları

\begin{tabular}{lcc}
\multicolumn{1}{c}{ Method } & \%60 oranında Eğitim & \%40 oranında Eğitim \\
\hline GoogLeNet (Xia et al. 2017) & $94.71 \pm 1.33 \%$ & $93.12 \pm 0.82 \%$ \\
\hline CaffeNet (Xia et al. 2017) & $96.24 \pm 0.56 \%$ & $95.11 \pm 1.22 \%$ \\
\hline VGG-16 (Xia et al. 2017) & $96.05 \pm 0.91 \%$ & $95.44 \pm 0.60 \%$ \\
\hline D-DSML-CaffeNet (Gong et al. 2017) & $96.64 \pm 0.68 \%$ & - \\
\hline MDDC (Qi et al. 2017) & $98.27 \pm 0.53 \%$ & - \\
\hline Fusion by Addition (Chaib et al. 2017) & $98.65 \pm 0.43 \%$ & - \\
\hline Önerilen Yöntem & $98.75 \pm 0.19 \%$ & $97.01 \pm 0.1 \%$ \\
\hline
\end{tabular}

\section{Sonuç ve Öneriler}

Bu araştırma makalesinde, WHU-RS19 veri setinde 19 sınıfa ait yüksek çözünürlüklü görüntülerin sınıflandırması için bir çalışma yapılmıştır. Bu çalışma aşağıda verilen adımlardan oluşmaktadır.

1. WHU-RS19 veri setindeki yüksek çözünürlüklü görüntülerin AlexNet, GoogleNet ve VGG-16 mimarilerine verilerek son katmanlarından özniteliklerinin çıkarılması,

2. ESA mimarilerinin elde edilen özniteliklerin birleştirilerek elde edilen toplam 3000 özniteliğin KBA algoritmasıyla tekrar 1000 özniteliğe indirgenmesi,

3. ESA mimarilerinden elde edilen özniteliklerin ayrı ayrı ve KBA ile indirgenmiş toplam 1000 adet özniteliğin kübik DVM ile sınıflandırılmasıdır.

$\mathrm{Bu}$ çalışmada VGG-16 mimarisinin DVM sınıflandırıcısı ile diğer mimarilere göre daha iyi performans gösterdiği görülmüştür. 3 mimariden elde edilen özniteliklerin KBA ile indirgenmesinin başarım oranında ciddi bir artışa sebep olduğu gözlemlenmiştir. Öznitelik sayısının fazla olması başarım oranını her zaman olumlu olarak etkilemediği görülmüştür. Ayrıca KBA'nın verimli öznitelikleri seçmede gayet başarılı olduğu saptanmıştır. Gelecekteki çalışmalarda, farklı ESA modelleri ile öznitelik indirgeme algoritmaları dikkate alınarak karşılaştırılmaları düşünülmektedir. Ayrıca, donanım altyapısı ile daha fazla işlem gücüne sahip büyük ve karmaşık veri setlerini kullanarak mevcut işin sınırlarını ortadan kaldırarak gerçek zamanlı uygulamaları çalıştırmak planlanmaktadır.

\section{Çıkar Çatışması}

Yazarlar tarafından herhangi bir çıkar çatışması beyan edilmemiştir.

\section{Kaynaklar}

Alom, M. Z., Taha, T. M., Yakopcic, C., Westberg, S., Sidike, P., Nasrin, M. S., ... \& Asari, V. K. (2018). The history began from AlexNet: a comprehensive survey on deep learning approaches. arXiv preprint arXiv:1803.01164.

Arel, I., Rose, D. C., \& Karnowski, T. P. (2010). Deep machine learning-a new frontier in artificial intelligence research. IEEE computational intelligence magazine, 5(4), 13-18.

Chaib, S., Liu, H., Gu, Y., \& Yao, H. (2017). Deep feature fusion for VHR remote sensing scene classification. IEEE Transactions on Geoscience and Remote Sensing, 55(8), 4775-4784.

Cheng, G., Han, J., Guo, L., Liu, Z., Bu, S., \& Ren, J. (2015). Effective and efficient midlevel visual elementsoriented land-use classification using VHR remote sensing images. IEEE Transactions on Geoscience and Remote Sensing, 53(8), 4238-4249.

Cheng, G., Han, J., Zhou, P., \& Guo, L. (2014). Multi-class geospatial object detection and geographic image classification based on collection of part detectors. ISPRS Journal of Photogrammetry and Remote Sensing, 98, 119-132.

Çalışkan, A. (2018). iki farkı bölge için uzaktan algılama yöntemlerine dayalı olarak ısı adaları ve şehirleşme analizi, Yüksek Lisans Tezi, İstanbul Aydın Üniversitesi fen bilimleri enstitüsü, İstanbul, 66 .

Göksu, Ö., \& Aptoula, E. (2018, May). Content based image retrieval of remote sensing images based on deep features. In 2018 26th Signal Processing and 
Communications Applications Conference (SIU) (pp. 1-4). IEEE.

Gong, Z., Zhong, P., Yu, Y., \& Hu, W. (2017). Diversitypromoting deep structural metric learning for remote sensing scene classification. IEEE Transactions on Geoscience and Remote Sensing, 56(1), 371-390.

Han, J., Zhang, D., Cheng, G., Liu, N., \& Xu, D. (2018). Advanced deep-learning techniques for salient and category-specific object detection: a survey. IEEE Signal Processing Magazine, 35(1), 84-100. Doi: 10.1109/MSP.2017.2749125

Huang, Z., Zhang, Y., Li, Q., Li, Z., Zhang, T., Sang, N., \& Xiong, S. (2019). Unidirectional variation and deep CNN denoiser priors for simultaneously destriping and denoising optical remote sensing images. International Journal of Remote Sensing, 40(15), 5737-5748.

Hu, Q., Wu, W., Xia, T., Yu, Q., Yang, P., Li, Z., \& Song, Q. (2013). Exploring the use of Google Earth imagery and object-based methods in land use/cover mapping. Remote Sensing, 5(11), 6026-6042.

Hu, F., Xia, G. S., Hu, J., \& Zhang, L. (2015). Transferring deep convolutional neural networks for the scene classification of high-resolution remote sensing imagery. Remote Sensing, 7(11), 14680-14707.

Khan, S., Islam, N., Jan, Z., Din, I. U., \& Rodrigues, J. J. C. (2019). A novel deep learning based framework for the detection and classification of breast cancer using transfer learning. Pattern Recognition Letters, 125, 1-6.

Krizhevsky, A., Sutskever, I., \& Hinton, G. E. (2012). Imagenet classification with deep convolutional neural networks. In Advances in neural information processing systems (pp. 1097-1105).

Lin, Y. L., \& Wei, G. (2005, August). Speech emotion recognition based on HMM and SVM. In 2005 international conference on machine learning and cybernetics (Vol. 8, pp. 4898-4901). IEEE.

Lv, Q., Dou, Y., Niu, X., Xu, J., \& Li, B. (2014, July). Classification of land cover based on deep belief networks using polarimetric RADARSAT-2 data.
In 2014 IEEE Geoscience and Remote Sensing Symposium (pp. 4679-4682). IEEE.

Mutlu, H. E. (2018). Hiperspektral görüntü ve lidar verisinin derin öğrenme ile sınıflandırılması (Yüksek Lisans Tezi, Hacettepe Üniversitesi Fen Bilimleri Enstitüsü, Ankara, 67.

Özyurt, F. (2020). A fused CNN model for WBC detection with MRMR feature selection and extreme learning machine. Soft Computing, 1-10.

Özyurt, F, Avcı, E. (2019). İmge Sınıflandırması için Yeni Öznitelik Çıkarım Yöntemi: Add-Tda Algısal Özet Fonksiyonu Tabanlı Evrişimsel Sinir Ağ (Add-TdaEsa). Türkiye Bilişim Vakfı Bilgisayar Bilimleri ve Mühendisliği Dergisi, 12 (1), 30-38.

Simonyan K, Zisserman A (2014) Very deep convolutional networks for large-scale image recognition. arXiv:1409.1556

Szegedy C, Liu W, Jia Y, Sermanet P, Reed S, Anguelov D, Rabinovich A (2015) Going deeper with convolutions. In: Proceedings of the IEEE conference on computer vision and pattern recognition, 1-9.

Qassim, H., Verma, A., \& Feinzimer, D. (2018). Compressed residual-VGG16 CNN model for big data places image recognition. In 2018 IEEE 8th Annual Computing and Communication Workshop and Conference (CCWC) (pp. 169-175). IEEE. Doi: 10.1109/CCWC.2018.8301729

Qi, K., Yang, C., Guan, Q., Wu, H., \& Gong, J. (2017). A multiscale deeply described correlatons-based model for land-use scene classification. Remote Sensing, 9(9), 917.

Tuncer, T., \& Ertam, F. (2019). Neighborhood component analysis and reliefF based survival recognition methods for Hepatocellular carcinoma. Physica A: Statistical Mechanics and its Applications, 123143.

Xia, G. S., Hu, J., Hu, F., Shi, B., Bai, X., Zhong, Y., ... \& Lu, $X$. (2017). AID: A benchmark data set for performance evaluation of aerial scene classification. IEEE Transactions on Geoscience and Remote Sensing, 55(7), 3965-3981. 
Yang, J., Guo, J., Yue, H., Liu, Z., Hu, H., \& Li, K. (2019). CDnet: CNN-Based Cloud Detection for Remote Sensing Imagery. IEEE Transactions on Geoscience and Remote Sensing.

Zhang, W., Tang, P., \& Zhao, L. (2019). Remote Sensing Image Scene Classification Using CNNCapsNet. Remote Sensing, 11(5), 494. 